\title{
Portfolioarbeit als Professionalisierungsgelegenheit
}

\author{
Sandra Ballweg (Bielefeld/Deutschland)
}

\section{Portfolioarbeit zwischen Theorie und Praxis}

In den letzten Jahren wurde viel über Portfolioarbeit geschrieben. Didaktische Publikationen weisen auf das große Potenzial des Instruments hin: für die Entwicklung von Selbststeuerung, als alternative Form der Leistungsbewertung oder zur Reflexion. Portfolioarbeit fügt sich gut in handlungs- und kompetenzorientierte Konzepte ein und weist eine hohe Passung mit aktuellen fremd- und zweitsprachendidaktischen Strömungen auf (vgl. Königs in diesem Band). Diese Annahmen beziehen sich sowohl auf das Europäische Sprachenportfolio (ESP) als auch auf offene Portfoliokonzepte.

In der Praxis ist Portfolioarbeit allerdings nicht flächendeckend angekommen, sie sei kaum umsetzbar, berichten Lehrende. So ist 20 Jahre nach dem großen Boom ernüchternd festzustellen, dass sich Portfolioarbeit trotz anfänglicher Begeisterung und vielversprechender Ansätze nicht durchgesetzt hat und weit hinter den - zugegebenermaßen recht hohen - Erwartungen zurückbleibt (vgl. dazu für das ESP Little et al. 2011 sowie die Beiträge von Kolb und Little in diesem Band).

Nicht selten hört man inzwischen von Wissenschaftler*innen, die sich mit Portfolioarbeit befassen, sei es nun aus schulpädagogischer oder fremd- und zweitsprachendidaktischer Perspektive, dass die Forschung ihren Beitrag zur Entwicklung von Portfolioarbeit geleistet habe, dass nun die Praxis nachziehen müsse, um zum Gelingen beizutragen. Aus der Praxis wiederum gibt es den Vorwurf, Portfolioarbeit sei an der Unterrichtsrealität vorbei konzipiert worden. Dabei ist beachtenswert, dass Portfolioarbeit als Lehr-/Lerninstrument - oder zumindest ihre Vorläufer - ja in der Praxis entstanden ist, wie etwa verschiedene ähnliche Formate 
in der Reformpädagogik oder writing folders in den 1950er Jahren in der Schreibdidaktik in Großbritannien. Erst später folgte die konzeptionelle Ausarbeitung des Instruments - und viel später dann empirische Forschung. Für das Europäische Sprachenportfolio als besondere Form eines Portfolios gilt allerdings, dass es als sprachenpolitisches Instrument entwickelt und tendenziell top down in den Unterricht implementiert wurde und wird. Schon in ihrer Entstehungsgeschichte ist Portfolioarbeit also schwer zwischen Theorie und Praxis zu verorten.

Aber warum hat es Portfolioarbeit trotz ihrer Ursprünge in der Unterrichtspraxis eben dort so schwer? Dafür gibt es zahlreiche Gründe, die sich gegenseitig bedingen. Sicher kollidiert die lernendenorientierte Arbeitsweise mit den gegebenen Bedingungen im Fremdsprachenunterricht an einer Regelschule oder auch in einem prüfungsvorbereitenden außerschulischen Unterricht. Auch für die Lernenden ist die Arbeitsweise ungewohnt, und häufig fehlt schlichtweg die Zeit. Die Liste lässt sich leicht fortführen. In diesem Beitrag möchte ich aber einen Aspekt in den Vordergrund rücken, der meines Erachtens noch nicht ausreichend Beachtung gefunden hat, nämlich die Herausforderungen, vor denen sich Lehrende vor allem bei der Einführung von Portfolioarbeit sehen (vgl. Little in diesem Band). Zu oft tappt die Portfoliodidaktik noch in die Methodenfalle, denn das, was Königs (2014: 68) für die sogenannten Alternativen Methoden der 1980er Jahren schreibt, gilt häufig auch für didaktische Instrumente wie Portfolioarbeit:

Die Auseinandersetzung mit diesen Methoden [hier: die sog. Alternativen Methoden, S.B.] fand in aller Regel vor dem Hintergrund der Frage statt, was diese Methoden denn für den Lernenden brächten. Viel seltener wurde gefragt, was die einzelnen Methoden für die Lehrperson bedeuteten. Zwar wurde darauf hingewiesen, [...] dass der Lehrer hier eine offensichtlich neue, ihm in der Regel nicht vertraute Rolle zu übernehmen habe. Aber insgesamt wurde doch davon ausgegangen, dass die Überzeugung, mit der Wahl der Methode aus Lernersicht die richtige Entscheidung getroffen zu haben, den Lehrenden ,nur' noch in die Rolle eines Erfüllungsgehilfen drängte: Die Aufgabe des Fremdsprachenlehrers ist es diesen methodischen Diskussionen zufolge, die jeweiligen Konzepte umzusetzen (Königs 2014: 168).

Wie in vielen Bereichen des Unterrichts hat auch in der Portfoliodidaktik phasenweise „die Methodenfixierung den Blick auf den Anteil der Lehrkräfte am Gelingen oder Misslingen von Unterricht [verstellt]" (Schart 2014: 36) und Lehrende zu den oben beschriebenen „Erfüllungsgehilfen“ gemacht. So kommt es nicht selten vor, dass ihnen vorgeworfen wird, Portfolioarbeit „nicht richtig“ umzusetzen. Andererseits wird aber auch auf ihre Überforderung hingewiesen, die durch Portfolioarbeit entsteht. Es besteht offenbar die Tendenz, entweder vom Pol der „omnipotente[n] Allzuständigkeit von Lehrern und Lehrerinnen“ auszugehen oder sich aber auf „entlastende[...] Unzuständigkeitserklärungen der pädagogischen 
Akteure gegenüber der Übermacht der äußeren Verhältnisse“ (Bastian \& Helsper 2000: 181) zurückzuziehen. Keine dieser Perspektiven bietet eine gute Grundlage für die tatsächliche Analyse der Situation, die der Ausgangspunkt für die Einführung von Portfolioarbeit sein muss.

In diesem Beitrag möchte ich der Frage nachgehen, wie sich Portfolioarbeit für Lehrende gestaltet und wie der Einsatz von Portfolios im Unterricht gerade durch die spezifischen Herausforderungen zur Professionalisierungschance für Lehrende werden kann. Damit greife ich den Zusammenhang zwischen Portfolioarbeit und Professionalisierung auf, konzentriere mich aber nicht auf Lehr- und Ausbildungsportfolios als reflexionsförderndes Instrument (vgl. z.B. Burwitz-Melzer sowie Michalak \& Ulrich in diesem Band). Vielmehr beziehe ich mich auf den strukturtheoretischen Ansatz, der Irritationen und Herausforderungen in einer Unterrichtssituation als Ausgangspunkt für Professionalisierung versteht. Um die Herausforderungen beschreiben zu können, vor denen Lehrende bei der Einführung von Portfolioarbeit stehen, werde ich zunächst einige ausgewählte Forschungsergebnisse zur Wahrnehmung von Portfolioarbeit umreißen (Abschnitt 2) und anhand einer Fallstudie die Sichtweisen und Aushandlungsprozesse einer Lehrerin im universitären Deutsch-als-Fremdsprache (DaF)-Unterricht bei der Einführung des Instruments sichtbar machen (Abschnitt 3). Anschließend möchte ich versuchen, Portfolioarbeit im Spannungsfeld unterrichtlichen Handelns zu verorten und die darin liegende Chance zur Professionalisierung zu diskutieren (Abschnitt 4), um abschließend kurz einige daraus erwachsende Perspektiven für die Portfolioarbeit zu skizzieren (Abschnitt 5). Mit Portfolioarbeit meine ich damit verschiedene Portfolioformen im Kontext von Fremd- und Zweitsprachenunterricht.

\section{Wahrnehmung von Portfolioarbeit: Stand der Forschung}

In Bezug auf die Wahrnehmung von Portfolioarbeit liegen aus den vergangenen Jahren und Jahrzehnten vor allem Erkenntnisse zur Perspektive der Lernenden vor. Dabei wird dem Instrument in einigen Studien eine hohe Akzeptanz attestiert (vgl. z.B. Bellingrodt 2011: 253f., Gläser-Zikuda \& Lindacher 2007: 199, Häcker 2007: 240). Als besonders positiv bewerten die Lernenden interessanterweise die Organisation von Lerninhalten (vgl. Häcker 2007: 239), ein Ziel, das aus portfoliodidaktischer Perspektive keine hohe Priorität hat, doch für die Lernenden von Bedeutung oder zumindest leicht zugänglich erscheint. Weiterhin positiv bewertet werden Möglichkeiten zu selbstbestimmtem Lernen und die Dokumentationsfunktion nach innen, also für die Lernenden selbst, sowie nach außen, für andere (vgl. González 2009: 378, Grittner 2009: 162-169).

Demgegenüber werden zahlreiche Aspekte kritisch eingeschätzt: In allen Studien wird einvernehmlich herausgearbeitet, wie nachteilig sich die hohe Zeit- und Arbeitsbelastung für die Lernenden, aber auch für die Lehrenden darstellt (vgl. z.B. 
Breuer 2009: 310f., Dinn 2017: 285, Häcker 2007: 239f., Flächer 2011: 120, Koçoğlu 2008: 3, Martinez Lirola \& Rubio 2009: 100). Auch die Schreibintensität der Aktivitäten empfinden die Lernenden als unangenehm (vgl. z.B. Häcker 2007: 240). Einige Studien geben Hinweise auf eine differenziertere Sichtweise: So scheinen Jungen die sprach- und schreibintensiven Aktivitäten, die Teil der Portfolioarbeit sind, eher abzulehnen als Mädchen (vgl. Häcker 2007: 240, Pollari 1997: 46) und bezogen auf Deutschland Hauptschüler*innen eher als Schüler*innen anderer Schultypen (vgl. Häcker 2007: 240). Besonders deutlich wird darüber hinaus eine starke Tendenz zur Überforderung bei den Lernenden sowie Verständnisschwierigkeiten und Verunsicherung (vgl. Ballweg 2015: 249f., Breuer 2009: 311, Häcker 2007: 271, Flächer 2011: 118). Die ungewohnte Arbeitsweise und der Einsatz als eine zusätzliche Aufgabe führen zu Ablehnung (vgl. Brouër 2007: 257-259), was nicht zuletzt damit zusammenhängt, dass vor allem Reflexionsaktivitäten als mühsam und unangenehm empfunden werden (vgl. Bräuer 2002: 31). Ein wesentliches - und naheliegendes - Bewertungskriterium der Lernenden neben dem Empfinden der Arbeitsweise als mehr oder weniger angenehm ist die Bewertung hinsichtlich des Nutzens für das Erreichen sprachlicher Lernziele, der für sie nicht immer erkennbar ist (vgl. Ballweg 2015: 267-271).

Spätestens an dieser Stelle kommt die Frage auf, wie genau mit dem Portfolio im jeweiligen Kontext gearbeitet wurde und unter welchen Rahmenbedingungen Unterricht stattfand, dass solche Wahrnehmungen des Instruments zustande kamen. Schließlich ist Portfolioarbeit kein Programm, das eine bestimmte Abfolge von Handlungsschritten nach sich zieht, sondern ein Instrument, das von Lehrenden unter den jeweiligen Rahmenbedingungen zielgruppenspezifisch genutzt werden muss, um jeweils sehr unterschiedliche Ziele zu verfolgen, von der Reflexion über kollaboratives Lernen bis zur Leistungsbewertung, um nur einige mögliche Ziele zu nennen. Bei der Betrachtung der Lernendenwahrnehmung ist also zu berücksichtigen, wie diese sich im Zusammenspiel von Zielen, Erwartungen, Vorerfahrungen und Lerngewohnheiten der Lernenden einerseits und der Ausgestaltung der Portfolioarbeit in der konkreten Lern- und Unterrichtssituation durch die Lehrperson andererseits konstituiert (vgl. Ballweg 2015: 312).

Die zentrale Rolle von Lehrenden bei der Portfolioarbeit wird daher völlig zu Recht angemahnt (vgl. z.B. Little et al. 2011) und in empirischen Studien herausgearbeitet (vgl. z.B. Ballweg 2015, Becker 2013, Bellingrodt 2011, Dinn in diesem Band). Leider liegen kaum belastbare Ergebnisse vor, wie genau sich diese gestaltet, aber einige Anforderungen an Lehrende, die aus den zuvor zitierten Studien hervorgehen, werde ich im Folgenden kurz umreißen.

$\mathrm{Zu}$ den gelingensförderlichen Maßnahmen zählen das Verdeutlichen des Nutzens des Instruments zum Erreichen sprachlicher Lernziele (vgl. Ballweg 2015: 267-271), Regelmäßigkeit bei der Arbeit mit dem Instrument (vgl. Bellingrodt 2011: 254, Flächer 2011: 118f., Martinez Lirola \& Rubio 2009: 100) sowie eine hohe „konzeptionelle Klarheit“, um nicht aus einem falsch verstandenen Bedürfnis nach Offenheit zu wenig steuernd einzugreifen (Häcker 2007: 282). Diese konzep- 
tionelle Klarheit im Sinne eines Durchdringens der verschiedenen Funktionen und Möglichkeiten des Instruments Portfolio und entsprechenden didaktischen Entscheidungen scheint bei den Lehrenden in den dazu vorliegenden Studien nicht immer in der erwarteten Form gegeben zu sein. So gibt es in Bezug auf das ESP Befunde, die verdeutlichen, dass Lehrende zwar die Dokumentationsfunktion kennen, ihnen aber die pädagogische Funktion weniger vertraut bis gänzlich unbekannt ist (vgl. Ballweg \& Stork 2008). Auch Dinn (2017: 284f.) beschreibt nach Interviews mit Lehrenden, dass sich ihnen einige Funktionen des ESP nicht erschließen und beispielsweise der Einbezug von Portfolios in den Unterricht, vor allem aber in die Leistungsbewertung, nicht gelingt. Die Lehrenden in Dinns Studie haben Mühe, adäquate Bewertungskriterien zu finden und befürchten, dass die Fremdbewertung durch die Lehrenden der Entwicklung der Selbstbewertungsfähigkeit der Lernenden entgegenwirkt.

Allerdings lässt sich hier schon erkennen, dass es sich nicht (vorrangig) um ein Versäumnis der Lehrenden handelt, sondern vielmehr strukturelle Probleme vorliegen, wenn Lehrende in einem Spannungsfeld zwischen institutionellen sowie curricularen Vorgaben und Lerngewohnheiten einerseits und portfoliodidaktischen Konzepten andererseits agieren (vgl. z.B. Breuer 2009: 307). So sind individuelle Portfoliogespräche und manche Reflexionsaktivitäten für die Lehrenden zeitlich kaum zu bewältigen und die individualisierte Leistungsbewertung steht einem an Standards orientierten Benotungszwang mit Ziffernnoten entgegen. Wie auch bei anderen lernendenorientierten Verfahren stellt sich also die Frage nach der Passung der Arbeitsweise mit curricularen Vorgaben und institutionellen Rahmenbedingungen im Regelunterricht (vgl. Königs 2010: 109 sowie in diesem Band). Die Einführung von Portfolioarbeit alleine in eine wenig kompatible Lernkultur hat sich als nicht umsetzbar erwiesen (vgl. Becker 2013, Kolb 2007, Kolb in diesem Band).

Anhand einer Einzelfallstudie versuche ich im nächsten Schritt, einen Einblick in die Aushandlungsprozesse einer Lehrperson zu geben, die Portfolioarbeit neu einführt und versucht, in dem skizzierten Spannungsfeld zu agieren.

\section{Portfolioarbeit aus der Perspektive einer Lehrerin: Ergebnisse einer Fallstudie}

\subsection{Untersuchungskontext und methodisches Vorgehen}

In einer Studie zur Arbeit mit Portfolios in einem universitären DaF-Kurs zur Förderung der Schreibfertigkeit (Ballweg 2015) habe ich eine Lehrerin und sieben Studierende ein Semester lang begleitet. Der Kurs (Niveau B2) umfasste neun dreistündige Sitzungen und beinhaltete u.a. die Gestaltung des fremdsprachlichen Schreibprozesses, Wortschatzarbeit und sprachliche Mittel sowie studienrelevante Textsorten wie beispielsweise Vorstufen zu Hausarbeiten und Mitschriften in Vor- 
lesungen. An dem Kurs nahmen 16 internationale Studierende teil, von denen sieben als Untersuchungsteilnehmer*innen zur Verfügung standen. Es handelte sich dabei um Studierende, vornehmlich in englischsprachigen Studiengängen in den Ingenieurwissenschaften, die sich erst seit kurzer Zeit in Deutschland aufhielten und aus China, Brasilien, Australien, Frankreich, Indien und dem Iran kamen.

Die Lehrerin verfügte über umfangreiche Unterrichtserfahrung, hatte aber kaum Vorerfahrungen in der Portfolioarbeit. Für die Einführung eines Portfolios hatte sie sich entschieden, weil sie von den Möglichkeiten für die Schreibförderung, besonders in Bezug auf das Schreiben verschiedener Textversionen, gehört hatte. In der gesamten Kooperation zeigte sie eine große Offenheit und ließ mich an ihren Überlegungen und auch Unsicherheiten teilhaben.

Eingesetzt wurde ein offenes Portfoliokonzept als begleitendes Entwicklungsportfolio sowie als Beurteilungsportfolio. Von Seiten der Lehrerin gab es kaum Vorgaben, weder zu Inhalten noch zur Medialität (eine Studentin führte das Portfolio in Papierform, die anderen digital). Vorgegebene Inhalte waren lediglich Texte in verschiedenen Überarbeitungsstadien sowie ein Lerntagebuch. In der ersten Sitzung erläuterte die Lehrerin: „, sie entscheiden natürlich ganz alleine was in ihr portfolio REINkommen soll“" (UB 1, 152-154). ${ }^{1}$ Viele der Fragen zur Kontextdefinition (z.B. Ziele, Rolle in der Leistungsbewertung), Kommunikation und Organisation, die Bräuer und Keller (2013: 268) als relevant bei bzw. vor der Einführung eines Portfolios auflisten, blieben zunächst ungeklärt und wurden im Kursverlauf eher als implizite Erwartungen an die Lernenden herangetragen.

Bei den erhobenen Daten handelte es sich um vier leitfadengestützte Interviews mit der Lehrerin sowie 20 Interviews mit Studierenden, die Aufzeichnung von 21 Unterrichtsstunden sowie von vier Portfoliogesprächen, die die Lehrerin jeweils mit zwei bis fünf Studierenden führte. Das Datenmaterial wurde in Anlehnung an GAT 2 in EXMARaLDA transkribiert und orientiert an der Grounded Theory nach Strauss und Corbin (vgl. z.B. 1996) interpretiert.

In diesem Beitrag werde ich die Daten aus den Interviews mit der Lehrerin sowie ihre Anleitung zur Portfolioarbeit neu betrachten, um herauszuarbeiten, welchen Herausforderungen sie sich gegenübersieht und welche Aushandlungsprozesse sichtbar werden. Neben der Gestaltung der Portfolioarbeit durch die Lehrerin stehen auch ihre Überzeugungen und Haltungen im Fokus, denen ein Einfluss auf das Handeln zugeschrieben wird.

\footnotetext{
${ }^{1}$ In der Beschreibung der Daten steht UB für Unterrichtsbeobachtung, die Zahl für die entsprechende Sitzung (UB 1 = Beobachtung der ersten Sitzung). Anschließend findet sich die Zeilenangabe aus dem Transkript. Die Abkürzung I_LP steht für das Interview der Lehrperson, die Zahl beantwortet, das wievielte Interview (1-4) es ist. Eine kurze Übersicht über relevante Transkriptionszeichen findet sich am Ende des Beitrags.
} 


\subsection{Zentrale Ergebnisse: Unsicherheit und Aushandlung}

Betrachtet man die Unterrichtsmitschnitte, stellt sich die Frage, welche Vorstellung von Portfolioarbeit die Lehrerin hat und man ist - ähnlich wie in der Studie von Dinn (2017; vgl. Abschnitt 2) - geneigt, zu behaupten, dass sie nicht mit allen Funktionen des Instruments vertraut ist. Betrachtet man die Erläuterungen aber genauer und zieht die Interviewdaten hinzu, ergibt sich ein anderes Bild. Es zeigt sich, dass beispielsweise die Widersprüche in der Anleitung der Portfolioarbeit Teil ihres intensiven Aushandlungsprozesses mit dem theoretischen Konzept vor dem Hintergrund ihrer Unterrichtspraxis sind.

Eine große Herausforderung, vor der die Lehrerin steht, sind die vielfältigen möglichen Funktionen eines Portfolios, die der geforderten konzeptionellen Klarheit (vgl. Abschnitt 2) im Weg stehen, zumal sich die Lehrerin diese Informationen ohne Fortbildung oder Austausch selbst erarbeitet hatte. Interessanterweise setzt sie bei der Wahl einer Funktion intuitiv auf eine, die in der Portfoliodidaktik in den letzten Jahren keine nennenswerte Rolle spielte, aber von den Lernenden (vgl. Abschnitt 2) als großen Nutzen von Portfolioarbeit wahrgenommen wird, nämlich die Organisation von Lerninhalten (I_LP I, 38-45). Diese Funktion wird auch von den Studierenden in dieser Studie bereitwillig angenommen. Aus ihrem Erfahrungswissen heraus wählt die Lehrerin also einen Schwerpunkt, der gut in ihren Unterricht passt, auch wenn es nicht das Konzept eines Portfolios ist, das man in Lehrbüchern findet. Zu Unstimmigkeiten kommt es dann, wenn sie in den Erklärungen von der Organisationsfunktion und von der Dokumentation nach außen spricht, die Arbeitsweise aber der eines Entwicklungsportfolios entspricht, das die individuelle Entwicklung, in diesem Fall der Schreibfertigkeit, zum Ziel hat, was sich zur Organisation von Lerninhalten oder als Nachschlagewerk weniger gut eignet. Hier beginnt dann ein Kreislauf, in dem durch Widersprüchlichkeiten in der Anleitung der Portfolioarbeit bei den Studierenden Unsicherheiten entstehen, die wiederum die Lehrerin verunsichern.

Auch wenn die Lehrerin durch Erklärungen und durch die Anlage des Unterrichts ein Verständnis von Portfolioarbeit vermittelt, ist ihre zentrale Überzeugung doch die, dass Portfolioarbeit konzeptionelle Offenheit bedeutet und maximale Offenheit eine Voraussetzung für Individualisierung ist. Sie nimmt weiter an, dass zu viele Erklärungen der Selbststeuerung entgegenstehen bzw. die Motivation mindern könnte. So sagt sie:

(.h) ich - äh. hatte $s o=n$ bisschen die befürchtung wenn ich denen gleich am anfang sage was was $\mathrm{ICH}$ unter dem portfolio verstehe oder denen ZU viel input gebe (mhm) dass die sich nicht mehr selber da dran setzen .. sondern dass die das ... wie ne ganz normale übung (I_LP II, 74-79).

Sie will Erklärungen nur geben, wenn die Studierenden „damit überhaupt nicht KLARkommen“ (I_LP I, 112-116). Im Unterricht drückt sich die Offenheit durch 
Vagheit und Komplexitätsreduktion aus, die vielleicht auch ein Indiz dafür sein kann, dass der Lehrerin selbst eine eher ungenaue Arbeitsdefinition zur Verfügung steht. So erläutert sie Portfolioarbeit in der ersten Unterrichtsstunde folgendermaßen:

aber wichtig ist dass das hier sozusagen ihre perSÖNliche ... sammel- ... reflexions- ... ähm . was auch immer mappe ist $-\mathrm{ja}^{6}$.. SIE gestalten die und SIE sammeln die dinger und heften die ab (UB 1, 258-263).

Schon in Bezug auf die Funktionen des Portfolios haben sich Widersprüchlichkeiten abgezeichnet. Diese zeigen sich auch in anderen Bereichen, beispielsweise wenn sich in einem Kurs zur Förderung der Schreibfertigkeit der Fokus immer weiter auf die Gestaltung des Portfolios verschiebt, bis zu dem Punkt, dass bei der abschließenden Leistungsfeststellung die Textqualität nicht mehr berücksichtigt wird. Das Portfolio nimmt immer mehr Raum ein, so dass die Lehrerin entscheidet, „die textschreiberei ein bisschen kürzer zu fassen“ und auf zu Hause zu verlagern, um in den Sitzungen mehr Zeit für die Arbeit am Portfolio zu haben (I_LP II, 335-341). ${ }^{2}$ Portfolioarbeit nimmt den größten Teil der Planungs- und Unterrichtszeit ein und bedeutet sowohl für die Studierenden als auch für die Lehrerin einen deutlich erhöhten Arbeitsaufwand, dem die Lehrerin nur gerecht werden kann, in dem sie andere Unterrichtsinhalte reduziert.

Weitere Widersprüchlichkeiten bestehen in den Rollen sowohl der Lehrerin als auch der Lernenden. Die Lehrerin nimmt wahr, dass sie sich zwischen Zurückhaltung und enger Betreuung, zwischen Prüferin und „kumpelhafter“ Begleitung (I_LP III, 220-315) verorten muss, was ihr große Mühe bereitet. Von den Lernenden erwartet sie, dass sie selbstgesteuert und eigenverantwortlich agieren, traut es ihnen aber nur begrenzt zu:

also hört sich jetzt vielleicht . gemein an dem kurs gegenüber dass ich denen nicht so viel zugetraut hab (.hh) aber ich hab/ ich hatte am ende wirklich die befürchtung dass die mehrheit nicht wirklich damit umgehen kann (I_LP IV, 194-198).

Hieran und an einigen weiteren Stellen im Datenmaterial wird die Schwierigkeit besonders deutlich, vor der die Lehrerin steht: Sie setzt ein Instrument im Unterricht mit der Befürchtung ein, dass die Studierenden daran scheitern könnten, begründet durch den Eindruck, dass sie Mühe mit dieser selbstgesteuerten Arbeitsweise haben. Im Kursverlauf versucht die Lehrerin immer wieder, den Studierenden die Verantwortung für ihren Lernprozess zu übertragen, die diese aber nicht annehmen (können) und an die Lehrerin zurückgeben.

\footnotetext{
${ }^{2}$ Vgl. hierzu den gain loss effect of portfolio use (Ballweg 2016), der besagt, dass Portfolioarbeit nicht nur ein Zugewinn für Unterricht und Lernen ist, sondern dass die Implementierung des neuen Instruments in der Regel auch bedeutet, bewährte Verfahren aufzugeben, Zeitressourcen zu investiert etc.
} 
Insgesamt ist das Handeln der Lehrerin von Unsicherbeiten und Befürchtungen geprägt, die sich vor allem darauf beziehen, dass sie Portfolioarbeit nicht erklären kann und die Studierenden sie eventuell nicht bewältigen werden. Sie spricht in Bezug darauf selbst mehrfach sogar von „Angst“. Befeuert werden die Befürchtungen dadurch, dass die Studierenden ihrerseits verunsichert und teilweise auch unzufrieden sind. $\mathrm{Da}$ die Lehrerin glaubt, nicht eingreifen zu dürfen, stellt die Angst vor dem Scheitern der Studierenden eine umso größere Belastung dar und macht sie hilflos und handlungsunfähig.

Auffällig ist, dass sich die Unsicherheit auf die Portfolioarbeit bezieht, nicht aber auf die ihr vertrauten Unterrichtsinhalte, z.B. Fragen der Grammatik-, Wortschatz- oder Schreibdidaktik. Hier fühlt sie sich sicher und ist im Unterricht weitaus expliziter in ihren Erklärungen. Das wirft die Frage auf, wie die bestehenden Kompetenzen, Erfahrungen und Sicherheiten als Ressource bei der Einführung neuer methodisch-didaktischer Elemente in den Unterricht genutzt werden und die durch Neues hervorgerufene Unsicherheiten reduziert werden können.

Ein besonderer Bereich ist jedoch die Leistungsbewertung, bei der die Lehrerin ihre große Unsicherheit zum Ausdruck bringt und am Ende gar auf die Bewertung der Texte verzichtet, obwohl sie durch äußere Vorgaben Noten vergeben muss. Es wird deutlich, dass sie bezüglich der Bewertung schriftlicher Texte schon vor der Einführung von Portfolioarbeit unsicher war, was in herkömmlichen Bewertungsverfahren über- oder umgangen werden konnte, indem Texte mit einer Ziffernnote versehen wurden. Durch die neue Form der Leistungsbewertung und den Anspruch der Lehrerin, die Note dieses Mal zu begründen, kommt sie in Erklärungsnot. Nicht das Portfolio verunsichert die Lehrerin an dieser Stelle, vielmehr wird eine zuvor vorliegende Unsicherheit durch die Besonderheiten der Portfolioarbeit zum Vorschein gebracht und verstärkt.

Die dargelegten Ergebnisse sind als Teil eines Einzelfalls zu verstehen, der sich durch zahlreiche individuelle, institutionelle und situative Besonderheiten konstituiert. Dabei ist zu beachten, dass das Handeln der Lehrerin auch durch die Tatsache beeinflusst wurde, dass es Teil einer empirischen Studie war. So entstanden vermutlich zusätzliche Reflexions- und auch Rechtfertigungsmomente durch die Interviews. Dennoch ergibt sich ein Einblick in die intensiven Aushandlungsprozesse dieser Lehrerin, die sich im Unterricht in einer Form niederschlagen, die auch in anderen Studien beobachtet wurden (größtmögliche Offenheit, geringe konzeptionellen Klarheit etc.). Es ist also anzunehmen, dass die Aushandlungsprozesse dieser Lehrerin bei aller Individualität Anteile haben, die denen anderer Lehrender durchaus ähneln.

Hierbei wurden verschiedene Dilemmata und Herausforderungen deutlich, nicht zuletzt, wie sehr die Lehrerin in ihren Überzeugungen verhaftet ist, obwohl sie sich zumindest teilweise auch als hinderlich darstellen. 


\section{Herausforderungen und Professionalisierungsgelegenheiten}

\subsection{Herausforderungen als Ausgangspunkt für Professionalisierung}

Anhand der Fallstudie wird sichtbar, dass sich die Lehrerin bemüht, Portfolioarbeit einzuführen und eine geeignete Vorgehensweise zu entwickeln, dass ihr aber an vielen Stellen entsprechende Ressourcen oder Möglichkeiten fehlen. Aus Sicht der Studierenden, der Lehrerin und in Bezug auf die Kursinhalte wurde die Portfolioarbeit eher negativ wahrgenommen. Warum lohnt es sich trotzdem, an Portfolios festzuhalten? Im Folgenden möchte ich betrachten, inwiefern die Lehrerin in der Fallstudie einen wertvollen Professionalisierungsschritt vollzieht und welche weiteren Maßnahmen nötig wären, um die Lehrerin zu unterstützen. Zu diesem Zweck beziehe ich aus strukturtheoretischer Sichtweise die Herausforderungen als Antinomien unterrichtlichen Handelns auf Portfolioarbeit, bevor ich eine berufsbiografische Sichtweise und das Konzept der Reflexiven Professionalität hinzuziehe.

Der strukturtheoretische Ansatz rückt die Rahmenbedingungen des Lehrer*innenhandelns in den Vordergrund und diskutiert Antinomie und Paradoxien von Unterrichtsgeschehen. Er fokussiert Handlungsprobleme von professionell Handelnden unter der Maxime struktureller Unsicherheit (vgl. Helsper 2011) und geht davon aus, dass die „Krise“, im Sinne der Unterbrechung von Routinen, z.B. durch Störungen jeder Art, im unterrichtlichen Handeln der Normalfall und Ausgangspunkt für Professionalisierung ist (vgl. Helsper 2011: 8). Die Einführung von Portfolioarbeit stellt demnach als Veränderung der Arbeitsweise eine Unterbrechung von Routinen auf mehreren Ebenen dar und bewegt sich nicht nur in den ohnehin gegebenen Antinomien unterrichtlichen Handelns, die ich im Folgenden umreißen werde, sondern verstärkt Spannungen durch die zum Regelunterricht im Widerspruch stehende Ausrichtung in besonderer Weise. Das gilt für Papierportfolios ebenso wie für elektronische Portfolios, wobei anzunehmen ist, dass in Lerngruppen, in denen bisher wenig mit elektronischen Medien gearbeitet wurde, die Einführung eines ePortfolios zu noch stärkeren Irritationen führen könnte.

Eine zentrale Antinomie unterrichtlichen Handelns, die in der Fallstudie bereits gut sichtbar wurde, ist die von Autonomie und Heteronomie, die Helsper (2000: 150f.) als Grunddilemma der Autonomieförderung bei noch bestehender Abhängigkeit und unter sozialen Zwängen beschreibt. Genau dieses unauflösbare Dilemma prägt die Reflexionen der Lehrerin im beschriebenen Kurs zur Schreibförderung, wenn sie Studierende zu Autonomie anleitet, diese sogar erwartet, dabei Scheitern befürchtet und gleichzeitig die anleitende und bewertende Instanz ist.

Das Spannungsfeld zwischen Anleitung auf der einen Seite und der Schaffung von Freiräumen auf der anderen Seite ist Gegenstand der sog. Symmetrie- bzw. Machtantinomie (vgl. Helsper 2000: 146). Nicht nur bei Portfolioarbeit, sondern in jeder Form von Unterricht ist die Beziehung zwischen Lehrenden und Lernenden durch ein komplexes System von Macht und Symmetrie gekennzeichnet. Bei stark 
lernendenorientierten Arbeitsweisen wird eine Neudefinition von Rollen vollzogen. Damit einher geht nicht nur eine ständige Neudefinition der eigenen Rolle der Lehrperson, sondern auch die Lernenden müssen die ihnen immer wieder neu zugewiesenen Rollen jeweils erkennen und annehmen, was häufig nicht geschieht (vgl. z.B. Ballweg 2015, Stork 2017). Eine besonders schwierige Form des Rollenwechsels findet in Bezug auf Symmetrie und Macht beim Übergang der Lehrenden von Lernbegleitung zu Leistungsbewertung statt (vgl. z.B. Breuer 2009: 307).

Leistungsbewertung ist geprägt von einer weiteren Antinomie, der Differenzierungsantinomie (vgl. z.B. Helsper 2000: 150f.): Auch hier verstärken Portfolioarbeit und andere methodisch-didaktische Ansätze, die auf Differenzierung, Individualisierung und Öffnung von Unterricht abzielen, bestehende Spannungsverhältnisse, denn sie arbeiten in Bezug auf Leistung verstärkt mit einer individuellen Bezugsnorm; curriculare und institutionelle Vorgaben setzen hingegen in der Regel eine sachliche Bezugsnorm an, orientiert an Lehrzielen und Sprachniveaus. So kann es vorkommen, dass Lernende große Entwicklungsschritte vollziehen, diese aber trotzdem ungenügend sind, wenn vorgegebene Lehrziele noch nicht erreicht werden konnten. Lehrende bewegen sich ständig in diesem Feld und verursachen nicht selten Brüche, die die Lernenden wiederum irritieren.

Das vermutlich größte Spannungsfeld liegt aber in der Tatsache, dass Portfolioarbeit mit Idealbildern von Lernenden, Lehrenden und Unterricht als „,implizite Betriebsprämisse“ (Häcker 2007: 281) operiert, also von dafür geschulten Lehrenden, großen zeitlichen Ressourcen und autonomen, motivierten Lernenden ausgeht (vgl. Ballweg 2015: 341f.). Idealvorstellungen liegen auch den leitenden Konzepten wie Individualisierung, Öffnung von Unterricht, Förderung von Lernendenautonomie, Reflexionsfähigkeit, kooperativem Lernen und z.T. der Entwicklung von Mehrsprachigkeit zugrunde, so dass Lehrende, Lernende und Unterrichtssituationen damit überfordert sind. Hier ist auf die Praxisantinomie (vgl. Helsper 2000: 145) zu verweisen, die sich auf die Forderung bezieht, mit theoretischen und wissenschaftlichen Erkenntnissen und Konzepten zu arbeiten, bei gleichzeitigem Wissen, dass eine Umsetzung in der Praxis nur schwer möglich ist. Daran anknüpfend ist die Antinomie von Rekonstruktion und Subsumtion relevant, die sich zwischen standardisierten Konzepten und fallbasierten Vorgehensweisen bewegt (vgl. Helsper 2000: 145). Manche portfoliodidaktischen Publikationen mit Rezeptcharakter können Lehrende zu einer standardisierten Vorgehensweise verleiten, so wie auch die Lehrerin in der Fallstudie versucht, Portfolioarbeit nach ihrem Verständnis einer empfohlenen Vorgehensweise umzusetzen statt einen eigenen, stärker den Bedingungen angepassten Weg zu finden.

Die Einordnung der in der vorgestellten Fallstudie sowie der in Abschnitt 2 umrissenen Ergebnisse empirischer Studien in das strukturtheoretische Konzept der Antinomien von Unterricht sollte dazu beitragen, den Eindruck, dass Portfolioarbeit in der Praxis ,nicht funktioniert“, besser greifbar zu machen. Portfolioarbeit, wie andere lernendenorientierte Ansätze auch, trifft gewissermaßen genau die wunden Punkte von Unterricht und verstärkt bestehende Spannungs- 
verhältnisse. Die Antinomien sollen allerdings nicht nur als Analyseinstrument des Scheiterns genutzt werden, denn anknüpfend an strukturtheoretische Sichtweisen bieten genau die entstehenden Irritationen und „Krisen“ eine Professionalisierungsgelegenheit.

\subsection{Professionalisierungsgelegenheiten}

Die Einführung von Portfolioarbeit mit allen Herausforderungen bietet eine Gelegenheit, gegebene Strukturen, das Instrument Portfolio und den eigenen Unterricht mit den jeweiligen Überzeugungen und Ressourcen zu reflektieren, und ist daher als Gelegenheit zur Professionalisierung zu verstehen. Unter Professionalisierung verstehe ich hier - eine große Diskussion stark vereinfachend - Prozesse des Professionell-Werdens und der zunehmenden Ausbildung von professioneller Handlungsfähigkeit sowie professioneller Identität, die von den individuellen Lehrenden ausgehen und gestaltet werden. Kennzeichnend sind also die individuellen, von den einzelnen Lehrenden initiierten, prozesshaften Integrationsprozesse von neuen Perspektiven und Verfahren, die von einem reflektierenden Habitus begleitet werden (vgl. zur Reflexion bei (angehenden) Lehrenden Burwitz-Melzer in diesem Band). Damit beziehe ich mich auf eine berufsbiografische Sichtweise unter Einbezug von kompetenzorientierten und strukturtheoretischen Ansätzen, verbinde also die drei zentralen in der Schulpädagogik genutzten Professionalisierungsansätze, die sich m.E. trotz ihrer unterschiedlichen Rhetorik gut zusammenführen lassen (vgl. dazu auch Gerlach 2019: 44).

Im Sinne des strukturtheoretischen Ansatzes besteht professionelles Handeln im Bewusstsein für Antinomien und einen reflektierten, balancierenden Umgang damit (vgl. Helsper 2000), als „gelassene Annahme der Krise“, verstanden als die Unterbrechung von Routinen, die „erst die Möglichkeit [impliziert], sich umfassend mit der Krisenkonstellation auseinandersetzen und sie reflektieren zu können" (Helsper 2001: 11). Professionalisierung wird in diesem Zusammenhang verstanden als die Entwicklung eines „doppelten Habitus“, der sowohl eine wissenschaftlich-reflexive Haltung als auch einen „Habitus des routinisierten, praktischen Könners“" (Helsper 2001: 13) beinhaltet, wobei letzterer notwendig ist, um überhaupt handlungsfähig zu bleiben. Nach Helsper (2000: 159; 2011: 11f.) ist zu dieser Entwicklung das fallrekonstruktive Arbeiten mit den skizzierten Antinomien in einem handlungsentlasteten Raum notwendig. Fallrekonstruktiv arbeitet auch die berufsbiografische Sichtweise, die strukturelle Spannungen ebenso wie Kompetenzorientierung mitdenkt, sie aber nicht in den Mittelpunkt stellt. Vielmehr konzentriert sie sich auf die individuelle professionelle Entwicklung von Lehrenden in ihrer Lehrendenpersönlichkeit. Professionalisierung ist in diesem Sinne nicht als standardisiertes Programm zu verstehen, sondern ein „veränderliches, kontinuierliches Entwicklungsproblem“ (Rossa 2018: 143, vgl. dazu auch Hericks 2006 und Terhart 2011), bei dem berufliche Anforderungen vor dem Hintergrund persönlicher, sozialer und biografischer Ressourcen betrachtet werden (vgl. Keller- 
Schneider 2010: 113). Dabei wird auch der Tatsache Rechnung getragen, dass Lehrende im Unterricht nicht nur aus einem explizit verfügbaren fachdidaktischen Wissen heraus handeln, sondern nicht selten auch ihre Intuition zum Einsatz kommt (vgl. Rossa 2018: 143). Leitend ist das Idealbild des reflective practitioner (Schoen 1983).

Im Rahmen dieses Beitrags stellt sich die Frage, was Lehrende brauchen, um in Bezug auf Portfolioarbeit ihre Professionalität weiterzuentwickeln. Hier wird deutlich, dass die Fortbildungsangebote, die in der Vergangenheit vielleicht die verbreitetste Maßnahme waren, um Portfolios in der Praxis zu implementieren, vor diesem Professionalisierungsverständnis wenig ertragreich sein können. Eine rezeptartige Vermittlung, wie Portfolioarbeit funktioniert, wird selten zu einem flexiblen, reflektierten Einsatz führen.

Hier möchte ich auf den Ansatz der Reflexiven Professionalität aus der Sozialen Arbeit (vgl. Dewe 2009) verweisen, aus dem ich Überlegungen zur Transformierbarkeit von unterschiedlichen Wissenstypen in Theorie und Praxis entleihe. Ausgangspunkt ist auch hier, dass ein einfacher Transfer von wissenschaftlichen Konzepten in die Praxis nicht möglich sein kann und damit Komplexität und Eigenlogik des Gegenstandsbereichs sowie der handelnden Akteur*innen vernachlässigt werden (vgl. Dewe 2009: 47-50). Vielmehr geht es um die Auflösung der Differenz zwischen

von Handlungs- und Entscheidungszwang entlastete[m] Theoretisieren und Forschen einerseits und dem stets situationsbezogenen, fallorientierten und unter hohem Handlungs- und Entscheidungsdruck stehenden professionellen Tun andererseits (Dewe 2009: 49f.).

Dass fallrekonstruktives Arbeiten, Aktionsforschung und forschendes Lernen hilfreiche Wege zur Überbrückung der Differenz von Theorie und Praxis (vgl. dazu z.B. Ohm \& Zörner 2019) und zur Entwicklung von Professionswissen als durch komplexe Prozesse neu entstehende Wissensform (vgl. Dewe 2009) darstellen, ist nicht neu, aber deshalb nicht weniger relevant. Dabei müssen (berufs)biografische Elemente dezidiert einbezogen werden. Nicht selten ist ein Portfolio das Instrument der Wahl zur Unterstützung dieser Prozesse (vgl. Burwitz-Melzer, Michalak \& Ulrich sowie Gavaldon in diesem Band).

Ein gelungenes Beispiel, wie die Annäherung an Portfolioarbeit und die Professionalisierung von Lehrenden geschehen kann, zeigt eine Aktionsforschungsstudie von Kristmanson et al. (2011), in der eine wechselnde Gruppe von fünf bis zehn Fremdsprachenlehrenden in Kanada ihre Arbeit mit dem Europäischen Sprachenportfolio beleuchtet. Eine zentrale Erkenntnis ist die, dass die Lehrenden im Laufe der Zeit eine differenziertere Haltung („philosophical stance") gegenüber dem Instrument entwickeln, die den Leitprinzipien einer demokratischen Pädagogik und autonomen Lernens folgt. Die sich entwickelnde Rolle der Lehrenden besteht vor allem in der Schaffung von Wahlmöglichkeiten und Lern- 
gelegenheiten, in der Ermöglichung und Begleitung von Lernen sowie in der Eröffnung neuer Perspektiven und Möglichkeiten. Zeit und Erfahrung scheinen dabei wesentliche Voraussetzungen zu sein, um das Instrument mit seinen unterschiedlichen Möglichkeiten zu begreifen und es entsprechend einzusetzen (vgl. Kristmanson et al. 2011: 59). Auch der intensive kollegiale Austausch und Raum für die Entwicklung einer forschenden Haltung sind als förderliche Faktoren zu nennen, Bedingungen, die Lehrende im Unterrichtsalltag nicht immer vorfinden. Gerade Angebote zur Vorbereitung, begleitenden Unterstützung und kollegialen Netzwerken, die für Lehrende bei der Portfolioarbeit von großer Bedeutung wären (vgl. Yilmaz \& Akcan 2012: 172), stellen bisher eher eine Ausnahme dar. In der Entwicklung von „durch Austausch-, Kooperations- und Entwicklungsbestrebungen geprägten communit[ies] of practice" (Riemer 2018: 133, Hervorh. im Origninal) von Lehrenden liegt ein großes Potenzial auch für die Portfolioarbeit.

In der Studie von Kristmanson et al. entsteht aus dem Zusammenspiel von Unterricht und Wissen über das Europäische Sprachenportfolio Interesse, das Instrument zu implementieren. Im weiteren Verlauf verbinden sich theoretische Konzepte, Unterrichtspraxis und empirische Forschung immer mehr. Forschung geht nicht der Implementierung voraus, sie erfolgt auch nicht, wenn diese abgeschlossen ist, sondern beides geht Hand in Hand.

Aber nicht nur in diesem Beispiel sind Theorie und Praxis eng verzahnt. Auch im hier vorgestellten Fallbeispiel ist die Arbeit der Lehrerin in hohem Maße von theoretischen Konzepten und empirischer Forschung geleitet, wenngleich in einer anderen Form: Sie arbeitet mit einem Verständnis eines Prozessportfolios, legt großen Wert auf Reflexion und Offenheit - und reichert dieses Theoriewissen mit ihren eigenen, von ihrer Intuition stark beeinflussten Unterrichtstheorien an, zum Beispiel dem Verständnis, dass Lernende ein Portfolio brauchen, um ihr Lernen zu organisieren. Hätte sie ein ähnliches Netzwerk sowie Möglichkeiten zu Reflexion und Austausch, also handlungsentlastete Räume (vgl. z.B. Helpser 2000: 159), zur Verfügung gehabt wie die Kolleginnen in Kanada, wäre vermutlich auch ihr Forschungs-/Erkundungsprojekt Portfolioeinsatz anders verlaufen. Die Entwicklung einer forschenden Haltung (vgl. dazu für den Fremdsprachenunterricht z.B. Schart 2014: 48) geschieht nicht von alleine und ist nicht voraussetzungsfrei, sondern braucht eben solche Netzwerke, Reflexionsmöglichkeiten sowie Zeiten und Räume, in denen keine Handlungen und Entscheidungen notwendig sind. Das stellt damit ein zentrales Desiderat für die Professionalisierung von Lehrenden bei der Portfolioarbeit dar.

\section{Perspektiven für Professionalisierung und Forschung}

Der Ausgangspunkt dieses Beitrags war die tendenziell unterschiedliche Wahrnehmung von Portfolioarbeit in Forschung einerseits und Unterrichtspraxis andererseits, und die Annahme, dass in den Spannungen zwischen Theorie und Praxis 
auch Chancen für die Professionalisierung von Lehrenden liegen. Was können die hier diskutierten Antinomien und Professionalisierungsgelegenheiten nun für die Portfolioarbeit, gewissermaßen für eine Portfoliogeneration 2.0, bedeuten?

Portfolioarbeit eignet sich in doppelter Hinsicht gut als Ausgangspunkt für Professionalisierung, einmal durch eine besonders starke Irritation unterrichtlicher Routinen, aber auch durch den Charakter des Instruments selbst, der Individualisierung und Reflexion zum Thema macht, so dass Lehrende diese Arbeitsweise nicht nur für ihre Lernenden einführen, sondern auch auf sich selbst anwenden und dabei einen reflektierenden Habitus ausbilden können, zum Beispiel indem sie selbst ein Lehrportfolio führen (vgl. dazu z.B. Bräuer 2016).

Es dürfte deutlich geworden sein, dass Lehrende durch die Einführung von Portfolioarbeit eine umfangreiche Professionalisierungsgelegenheit für sich selbst schaffen, dass es aber andere Bedingungen braucht, um diese zu nutzen. Punktuelle Fortbildungen, die ein Instrument vorstellen und Lehrende damit in die Umsetzung entlassen, reichen nicht aus, um dazu Professionswissen zu entwickeln und die berufliche Handlungsfähigkeit sowie professionelle Identität nachhaltig weiterzuentwickeln. Fortbildung muss individueller und begleitend gestaltet sein, mit der Bildung einer community of practice einhergehen und die Verzahnung von Theorie und Praxis anlegen. Dabei sollten Ansätze von Forschendem Lernen, Aktionsforschung und fallrekonstruktivem Arbeiten genutzt werden und Freiräume zur Reflexion entstehen, um den Aufbau von Professionswissen, den Einbezug von Erfahrungswissen sowie die Weiterentwicklung der professionellen Identität der Lehrperson zu ermöglichen. Aber auch hier können wir von der Schulpädagogik profitieren, die uns daran erinnert, dass sich das Bestreben nach Professionalisierung und Reflexivität nicht in überhöhten Erwartungen und in Idealismus niederschlagen sollte, sondern ein pragmatisch-realistischer Zugang angestrebt werden muss (vgl. Häcker 2019: 83f.).

Nur durch verbesserte Bedingungen ist es möglich, dass Lehrende kompetent und selbstbewusst Entscheidungen bezüglich der Einführung und Umsetzung von Portfolioarbeit treffen und diese so ausgestalten, dass es den Bedürfnissen der Lernenden entspricht. ${ }^{3}$

Für die empirische Forschung bedeuten die hier angestellten Überlegungen, neben die Erforschung von gelungenen Portfoliokonzepten unter guten Bedingungen noch stärker als bisher auch solche Erhebungskontexte zu stellen, in denen Portfolioarbeit unter widrigen Umständen eingeführt oder umgesetzt wird, denn es hat sich gezeigt, dass die jeweiligen strukturellen Besonderheiten einen großen Einfluss auf die Ausgestaltungsmöglichkeiten haben. So unterscheiden sich die Ergebnisse beispielsweise in Grittners (2009) Studie, die an einer reformpäda-

\footnotetext{
3 Zu diskutieren wäre im Übrigen auch in Bezug auf Portfolioarbeit die besondere Situation von DaF- und DaZ-Lehrenden (vgl. dazu Funk 2018), die sich durch eine sehr heterogene Ausbildungssituation und unterschiedliche Zugangsmöglichkeiten zu Fort- und Weiterbildung von der anderer Fremdsprachenlehrender im deutschsprachigen Raum unterscheidet.
} 
gogisch orientierten Grundschule erhoben wurden, in vielerlei Hinsicht von denen aus Regelschulen oder aus der vorgestellten Fallstudie aus dem universitären Kontext. Zur Erforschung der Einsatzbedingungen und -strukturen bieten sich Ansätze an, die das komplexe Zusammenspiel, die Bedingungen und Einflussfaktoren sowie die Veränderungsprozesse in den Blick nehmen. Ansätze von Forschendem Lernen und Aktionsforschung stellen dabei wichtige Möglichkeiten dar, nicht nur für die Erforschung von Portfolioarbeit, sondern auch für die Professionalisierung von Lehrenden.

Festzuhalten bleibt, dass Portfolioarbeit nur dann sinnvoll sein kann, wenn bei ihrer Einführung die jeweiligen Bedingungen berücksichtigt werden. Damit muss auch Portfolioarbeit in jedem einzelnen Fall anders aussehen, ein Gedanke, der in der Portfoliodidaktik nicht neu ist, der aber bei einer Neuauflage des Europäischen Sprachenportfolios leitend sein könnte. Portfolioarbeit kann nur dann ernsthaft lernendenorientiert und autonomiefördernd sein, wenn Freiräume und Gestaltungsspielräume vorhanden sind, in denen sich auch längerfristig eine entsprechende Lernkultur entwickeln kann und Lehrende Reflexions- und Entwicklungsräume finden.

\section{Dank}

Für ihre wertvollen Kommentare und die hilfreichen Gespräche zu den ersten Ideen und frühen Fassungen dieses Beitrags danke ich Claudia Riemer, Udo Ohm, Gerd Bräuer und David Gerlach ganz herzlich. Mein Dank geht auch an die Lehrerin, die mich an ihrem Unterricht und an ihren Gedanken dazu Anteil haben ließ.

\section{Verwendete Transkriptionszeichen}

$\begin{array}{ll}\cdot & \text { Sprechpause bis 1 Sekunde } \\ . . & \text { Sprechpause bis 2 Sekunden } \\ = & \text { Verschleifungen/schneller Anschluss } \\ \text { SILbe (Großschreibung) } & \text { starke Betonung einer Silbe } \\ \text { Silbec } & \text { steigende Intonation } \\ \text { Silbe, } & \text { fallende Intonation } \\ \text { Silbe- } & \text { schwebende Intonation } \\ / & \text { Wortabbruch } \\ (. h),(. h h) & \text { einatmen, je nach Dauer } \\ (\text { h) },(\mathrm{hh}) & \text { ausatmen, je nach Dauer }\end{array}$




\section{Literatur}

Ballweg, Sandra (2015): Portfolioarbeit im Fremdsprachenunterricht. Eine empirische Studie zu Schreibportfolios im DaF-Unterricht. Tübingen: Narr.

Ballweg, Sandra (2016): Portfolios as a means of developing and assessing writing skills. In: Göpferich, Susanne; Neumann, Imke (Hrsg.): Developing and Assessing Academic and Professional Writing Skills. Frankfurt/M. u.a.: Lang, 143-172.

Ballweg, Sandra; Stork, Antje (2008): DaF-Lehrende und das Europäische Sprachenportfolio. In: InfoDaF. Informationen Deutsch als Fremdsprache 4, 390-400.

Bastian, Johannes; Helsper, Werner (2000): Professionalisierung im Lehrberuf. Bilanzierung und Perspektiven. In: Bastian, Johannes; Helsper, Werner; Reh, Sabine; Schelle, Carla (Hrsg.): Professionalisierung im Lebrerberuf. Opladen: Leske + Budrich, 167-192.

Baumert, Jürgen; Kunter, Mareike (2011): Das Kompetenzmodell von COACTIV. In: Kunter, Mareike; Baumert, Jürgen; Blum, Werner; Klusmann, Uta; Krauss, Stefan; Neubrand, Michael (Hrsg.): Professionelle Kompetenz von Lehrkräften. Ergebnisse des Forschungsprogramms COACTIOV. Münster/New York: Waxmann, 29-53.

Bellingrodt, Lena C. (2011): ePortfolios im Fremdsprachenunterricht. Empirische Studien zur Förderung autonomen Lernens. Frankfurt/M. u.a.: Lang.

Bräuer, Gerd (2002): Experimentieren mit einer Methode und Arbeiten am Text: Reformen durch Portfolios. In: ide. Informationen zur Deutschdidaktik 1, 25-34.

Bräuer, Gerd 2014. Das Portfolio als Reflexionsmedium für Lehrende und Studierende. Opladen u.a.: Budrich.

Bräuer, Gerd; Keller, Stefan (2013): Elektronische Portfolios als Katalysator für Studium und Lehre. In: Koch-Priewe, Barbara; Leonhard, Tobias; Pineker, Anna; Strörtländer, Jan Christoph (Hrsg.): Portfolio in der LehrerInnenbildung. Konzepte und empirische Befunde. Bad Heilbrunn: Klinkhardt, 265-275.

Breuer, Angela C. (2009): Das Portfolio im Unterricht. Theorie und Praxis im Spiegel des Konstruktivismus. Münster u.a.: Waxmann.

Brouër, Birgit (2007): Portfolios zur Unterstützung der Selbstreflexion - Eine Untersuchung zur Arbeit mit Portfolios in der Hochschullehre. In: GläserZikuda, Michaela; Hascher, Tina (Hrsg.): Lermprozesse dokumentieren, reflektieren und beurteilen. Lerntagebuch und Portfolio in Bildungsforschung und Bildungspraxis. Bad Heilbrunn: Klinkhardt, 235-265.

Dewe, Bernd (2009): Reflexive Professionalität: Maßgabe für Wissenstransfer und Theorie-Praxis-Relationierung im Studium der Sozialarbeit. In: Riegler, Anna; Hojnik, Sylvia; Posch, Klaus (Hrsg.): Soziale Arbeit zwischen Profession und Wissenschaft. Vermittlungsmöglichkeiten in der Fachhochschulausbildung. Wiesbaden: VS Research, 47-63. 
Dinn, Julia (2017): Kompetenzorientierte Leistungsermittlung: Welche Rolle spielt das Portfolio? In: Appel, Joachim; Jeuk, Stefan; Mertens, Jürgen (Hrsg.): Sprachen lehren. Baltmannsweiler: Schneider Hohengehren, 279-288.

Flächer, Tina (2011): Portfolioarbeit im gymnasialen Fremdsprachenunterricht. Themenorientierter Unterricht mit dem Europäischen Portfolio der Sprachen. Frankfurt/M. u.a.: Lang.

Funk, Hermann (2018): Deutsch als Fremd- und Zweitsprache als Vorreiter und Nachzügler einer Professionalisierung von Fremdsprachenlehrkräften $-\mathrm{zu}$ Unterschieden und gemeinsamen Herausforderungen. In: Burwitz-Melzer, Eva; Riemer, Claudia; Schmelter, Lars (Hrsg.): Rolle und Professionalität von Fremdsprachenlehrpersonen. Arbeitspapiere der 38. Frühjabrskonferenz zur Erforschung des Fremdsprachenunterrichts. Tübingen: Narr, 54-66.

Gerlach, David (2019): Second-order (?) Language Teachers. Rekonstruktionen professioneller Handlungspraxis und Ausbildungsdidaktik von Lebrerbildner/innen im Vorbereitungsdienst angehender Fremdsprachenlehrpersonen. Habilitationsschrift. Philipps-Universität Marburg.

Gläser-Zikuda, Michaela; Lindacher, Tanja (2007): Portfolioarbeit im Unterricht praktische Umsetzung und empirische Überprüfung. In: Gläser-Zikuda, Michaela; Hascher, Tina (Hrsg.): Lernprozesse dokumentieren, reflektieren und beurteilen. Lerntagebuch und Portfolio in Bildungsforschung und Bildungspraxis. Bad Heilbrunn: Klinkhardt, 189-204.

González, Jesús Á. (2009): Promoting student autonomy through the use of the European Language Portfolio. In: ELT Journal 4, 373-382.

Grittner, Frauke (2009): Leistungsbewertung mit Portfolio in der Grundschule. Eine mehrperspektivische Fallstudie aus einer notenfreien sechsjährigen Grundschule. Bad Heilbrunn: Klinkhardt.

Häcker, Thomas (2007): Portfolio: Ein Entwicklungsinstrument für selbstbestimmtes Lernen. Eine explorative Studie zur Arbeit mit Portfolios in der Sekundarstufe I. 2. Aufl. Baltmannsweiler: Schneider Hohengehren.

Häcker, Thomas (2019): Reflexive Professionalisierung. Anmerkungen zu dem ambitionierten Anspruch, die Reflexionskompetenz angehender Lehrkräfte umfassend zu fördern. In: Degeling, Maria; Franken, Nadine; Freund, Stefan; Greiten, Silvia; Neuhaus, Daniela; Schellenbach-Zell, Judith (Hrsg.): Herausforderung Kohärenz: Praxisphasen in der universitären Lehrerbildung. Bildungswissenschaftliche und fachdidaktische Perspektiven. Bad Heilbrunn: Klinkhardt, 81-96.

Helsper, Werner (2000): Antinomien des Lehrerhandelns und die Bedeutung der Fallrekonstruktion - Überlegungen zu einer Professionalisierung im Rahmen universitärer Lehrerausbildung. In: Cloer, Ernst; Klika, Dorle; Kunert, Hubertus (Hrsg.): Welche Lehrer braucht das Land? Notwendige und mögliche Reformen der Lebrerbildung. Weinheim: Juventa, 142-177. 
Helsper, Werner (2001): Praxis und Reflexion. Die Notwendigkeit einer „doppelten Professionalisierung“ des Lehrers. In: Journal für Lehrerinnen- und Lehrerbildung 3, 7-15.

Helsper, Werner (2011): Lehrerprofessionalität - der strukturtheoretische Professionsansatz zum Lehrberuf. In: Terhart, Ewald; Bennewitz, Hedde; Rothland, Martin (Hrsg.): Handbuch der Forschung zum Lebrerberuf. Münster et al.: Waxmann, 149-170.

Hericks, Uwe (2006): Professionalisierung als Entwicklungsaufgabe. Rekonstruktion zur Berufseingangsphase von Lehrerinnen und Lehrern. Wiesbaden: VS Verlag für Sozialwissenschaften.

Keller-Schneider, Manuela (2010): Entwicklungsaufgaben im Berufseinstieg von Lehrpersonen. Beanspruchung durch berufliche Herausforderungen im Zusammenhang mit Kontext- und Persönlichkeitsmerkmalen. Münster: Waxmann.

Koçoğlu, Zeynep (2008): Turkish EFL student teachers' perception on the role of electronic portfolios in their professional development. In: The Turkish Online Journal of Educational Technology 3, 1-9.

Königs, Frank G. (2010): Wie ernst müssen wir die Lernerorientierung nehmen? Oder: Warum Normenkonflikte im Fremdsprachenunterricht unausweichlich sind und wie wir damit umgehen können. In: Dose, Stefanie; Götz, Sandra; Brato, Thorsten (Hrsg.): Norms in educational linguistics. Frankfurt/M. u.a.: Lang, 101-115.

Königs, Frank G. (2014): War die Lernerorientierung ein Irrtum? Der Fremdsprachenlehrer im Kontext der Sprachlehrforschung. In: Fremdsprachen lernen und lehren 43/1, 67-80.

Kolb, Annika (2007): Portfolioarbeit. Wie Grundschulkinder ibr Sprachenlernen reflektieren. Tübingen: Narr.

Kristmanson, Paula L.; Lafargue, Chantal; Culligan, Karla (2011): From action to insight: A professional learning community's experiences with the European Language Portfolio. In: The Canadian Journal of Applied Linguistics 2, 53-67.

Little, David; Goullier, Francis; Hughes, Gareth (2011): The European Language Portfolio. The story so far (1991-2011). Strasbourg: Council of Europe.

Martinez Lirola, María; Rubio, Fernando (2009): Students' beliefs about portfolio evaluation and its influence on their learning outcomes to develop EFL in a Spanish context. In: International Journal of English Studies 1, 91-111.

Ohm, Udo; Zörner, Anika (2019): Gelegenheiten Forschenden Lernens im Fachgebiet Deutsch als Zweitsprache im Praxissemester. Theoretische Modellierung und empirische Analysen auf der Basis narrativer Interviews. Herausforderung Lebrer_innenbildung, 2/2, 124-154. doi:10.4119/UNIBI/hlz-134 [28.04.2019]. 
Pollari, Pirjo (1997): Could portfolio assessment empower EFL learners?

Portfolios in the teaching of English as a foreign language in Finnish upper secondary school. In: Huhta, Ari; Kohonen, Viljo; Kurki-Suonio, Liisa; Luoma, Sari (Hrsg.): Current Developments and Alternatives in Language Assessment.

Jyvaskyla: University of Jyvaskyla, 37-68.

Riemer, Claudia (2018): Die Rolle und Professionalität von DaF-/DaZ-

Lehrer*innen. In: Burwitz-Melzer, Eva; Riemer, Claudia; Schmelter, Lars

(Hrsg.): Rolle und Professionalität von Fremdsprachenlehrpersonen. Arbeitspapiere der 38.

Frübjahrskonferenz zur Erforschung des Fremdsprachenunterrichts. Tübingen: Narr, 131-142.

Rossa, Henning (2018): Die Professionalität der Fremdsprachenlehrperson aus der Innensicht: Wissen, Erfahrungen und beliefs. In: Burwitz-Melzer, Eva; Riemer, Claudia; Schmelter, Lars (Hrsg.): Rolle und Professionalität von

Fremdsprachenlehrpersonen. Arbeitspapiere der 38. Frübjahrskonferenz zur Erforschung des Fremdsprachenunterrichts. Tübingen: Narr, 143-151.

Schart, Michael (2014): Die Lehrerrolle in der fremdsprachendidaktischen Forschung: Konzeptionen, Ergebnisse, Konsequenzen. In: Fremdsprachen lernen und lehren 43/1, 37-50.

Schoen, Donald (1983): The reflective practitioner. How professionals think in action. New York: Basic books.

Stork, Antje (2017): Lernende im Dialog: Lerntagebücher im Fremdsprachenunterricht. http://geb.unigiessen.de/geb/volltexte/2017/13145/pdf/GiFon_10.pdf [01.03.2019].

Strauss, Anselm L.; Corbin, Juliet (1996): Grounded Theory. Grundlagen qualitativer Sozialforschung. Weinheim: Beltz.

Terhart, Ewald (2011): Lehrerberuf und Professionalität: Gewandeltes

Begriffsverständnis - neue Herausforderungen. In: Zeitschrift für Pädagogik 57. Beiheft, 202-224.

Yilmaz, Sinem; Akcan, Sumru (2012): Implementing the European Language

Portfolio in a Turkish context. In: ELT Journal 2, 166-174. 